\title{
Ridge Linking Using an Adaptive Oriented Mask Applied to Plant Root Images with Thin Structures
}

\author{
Talita Perciano ${ }^{1, \star}$, Roberto Hirata Jr. ${ }^{1}$, and Lúcio André de Castro Jorge ${ }^{2}$ \\ 1 Instituto de Matemática e Estatística, Universidade de São Paulo, \\ São Paulo, SP 05508-090, Brazil \\ \{talitap, hirata\}@ime.usp.br \\ 2 CNPDIA, Embrapa Instrumentação Agropecuária, São Carlos, \\ SP 13560-970, Brazil \\ lucio@cnpdia.embrapa.br
}

\begin{abstract}
A ridge linking algorithm and its application to plant root images is presented. The idea is an improvement of an edge linking algorithm where we explore local directional information. The experimental results show that we obtain good connections between ridge segments which tends to maintain the connectivity of structures like plant roots.
\end{abstract}

Keywords: Ridge linking, edge linking, ridge detection, image segmentation.

\section{Introduction}

Ridge detection, as well as edge detection, is a fundamental problem in image processing and computer vision. The detection must have a good quality in order to extract useful information. Ridge or edge detectors often provide noncontiguous ridges/edges, i.e., maps with many discontinuities and, therefore, without desired connected contours. In order to deal with this problem and to form connected ridges/edges, a linking step must be done.

Edge linking has been studied for many years being very close to ridge linking. A known algorithm is SEL (Sequential Edge Linking), proposed in 1985 by Eichel and Delp 314. It is a sequential search algorithm for edge detection and linking that uses a random field and a random Markov chain to model the problem. It has been improved later by using basically the same model but adding a multiresolution idea [2]. Because of the model used, many parameters must be set to obtain acceptable results.

In [8, a two-steps algorithm was proposed. It uses distance, direction and magnitude information of edge segments to build a weighting system to link the edges. Other algorithms are based on direction, local information around ending

^ Thanks to FAPESP, CNPq and CAPES for funding.

I. Bloch and R.M. Cesar, Jr. (Eds.): CIARP 2010, LNCS 6419, pp. 378 385, 2010.

(C) Springer-Verlag Berlin Heidelberg 2010 
points and function cost minimization [6/73]. The algorithm proposed in 13 makes some improvements to these methods related to the measurement of the edge direction and the distance used in the cost function for edge linking. The measurement of the edge direction is more precise, incorporating all possible directions between $0^{\circ}$ and $360^{\circ}$ degrees. The geodesic distance is used to measure the proximity between two ending points, in order to use the intensity information. We propose other improvements in this algorithm related to the edge direction and the mask used during the process. We show that the quality of the linked edges can be improved using an adaptive mask related to the local main direction around an ending point. This adaptive mask avoids unnecessary connections between ending points. Besides, it also avoids connections that are out of the global and local directional contexts of the image.

In the literature, other edge linking methods can be found using concepts of mathematical morphology and connectionist models [11], combining local and global measures for edge point linking 910. Finally, associated with the edge linking issue, edge grouping methods can also be found [12]. However, edge grouping aims to detect salient structures and boundaries, rather than improving the edge detection by connecting short edges.

The remainder of this paper is organized as follows. In Section 2, we describe the concepts and techniques used in the proposed method. In Section 3 , we show some experimental results. The conclusions in Section 4 synthesize the results of the paper and depict possible future works.

\section{Methodology}

The linking process of the proposed method is based on the following general steps: (1) find the ending points of the ridge/edge map, (2) select the appropriate candidate linking points for each ending point using an adaptive directional mask and (3) select the linking point using information of ridge direction and geodesic distance.

In our approach the ridges of the images are calculated combining the Canny edge detector applied directly to the original image (we do not use the gradient image) and a surround suppression proposed by [5].

This section describes the adaptive directional mask approach (the main modification over the existing methods) used by the proposed algorithm along with a review of the concepts used from the existing edge linking algorithms.

\subsection{Adaptive Directional Mask}

Among the existing linking approaches that use neighborhood information, the common used masks have fixed shapes and sizes given by the user. The directional local information is used only when deciding which candidate linking point should be connected with an ending point. We propose an additional strategy to 
explore this information to suppress some of the candidates. We use an orientation histogram to adapt a rectangular or a conical mask (shape and size chosen by the user) with the local directional context of the image.

Figure 1illustrates this process. One particular ending point is being analyzed (red one) during the process. In Figure 1(a) a rectangular mask is represented in gray and the candidate linking points in black. Figure 1(b) presents the ridge map for the analyzed window. In order to find the local main direction, a ridge orientation histogram is calculated for this window. The orientation with the highest frequency is chosen to be the orientation of the mask. In this particular case, the diagonal orientation was chosen. Figure 1(c) presents the rotated mask and Figure $1(\mathrm{~d})$ the final candidate linking points.

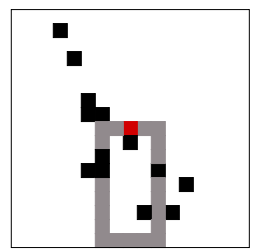

(a) Linking candidates.

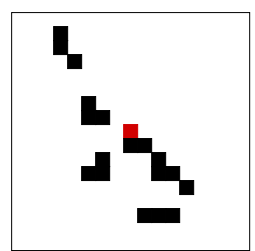

(b) Edges window.

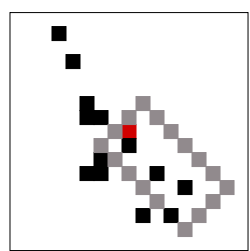

(c) Rotated mask.

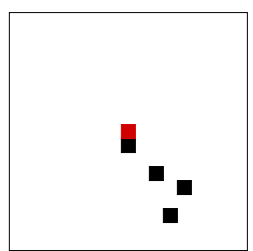

(d) Final linking candidates.

Fig. 1. Example of the mask rotation depending on local directional histogram

In a particular case that, for instance, two orientations have (almost) the same frequency in the histogram, the mask is rotated in both directions and all the candidate linking points in both cases are chosen. The orientations taken into account in the histogram depend on the edge detector used. The same orientations given by the detector will be used for the histogram.

In the following, we explain how to choose the point among the candidates to be connected to the ending point.

\subsection{Ridge Direction and Geodesic Distance}

After the suppression step, the remaining candidates are analyzed using the ridge/edge direction approach and geodesic distance proposed in [13.

Figure 2 illustrates the ridge/edge direction calculation. There is a portion of ridge/edge (ones), the ridge's/edge's end point $P_{e}$ (gray square) and some candidates to the continuation of the ridge/edge (two isolated points at the border). For the ending point $P_{e}$ all the ridge/edge points connected to it are used to fit a line $l$. The vector $\vec{d}_{c}$ represents the direction from the centroid of these points $P_{c}$ (gray circle) to $P_{e}$. Between the two possible directions of $l$, the one closest to $\vec{d}_{c}$ is chosen as the ridge/edge direction of $P_{e}$, i.e., $\vec{d}_{1}$. 


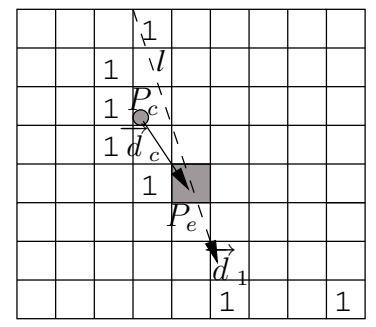

Fig. 2. Edge direction calculation by 13

This approach has the advantage that all the orientations from $0^{\circ}$ to $360^{\circ}$ are possible, rather than a discrete number of orientations.

The geodesic distance also proposed by 13 is used by our algorithm. The distance, $D_{g}\left(p_{i}, p_{j}\right)$, between two pixels $p_{i}$ and $p_{j}$ of an image $I$ is defined as the minimum path between these two pixels. Let us consider a path $P=\left\{p_{1}, p_{2}, \ldots, p_{n}\right\}$, where $p_{i}$ and $p_{i+1}$ are connected neighbors and $i \in\{1,2, \ldots, n-1\}$. The length of $P$ is

$$
l(P)=\sum_{i=1}^{n-1} d_{N}\left(p_{i}, p_{i+1}\right) .
$$

Thus, particular geodesic distances can be derived depending on the neighbors connection and the distance between neighbors. In this work, pixels are eight connected and $d_{N}\left(p_{i}, p_{i+1}\right)=\left|I\left(p_{i}\right)-I\left(p_{i+1}\right)\right|$. Using the geodesic distance, we are able to consider the information of the intensities of the pixels, rather than just their coordinates as when using the Euclidean distance.

For the evaluation of the candidate linking points, the following cost function is used:

$$
H\left(P_{e}, P_{c}\right)=\frac{1}{D_{g}\left(P_{e}, P_{c}\right) \cdot \Theta\left(P_{e}, P_{c}\right)},
$$

where $D_{g}\left(P_{e}, P_{c}\right)$ is the geodesic distance between $P_{e}$ and $P_{c}$ and $\Theta\left(P_{e}, P_{c}\right)$ is the angle between $\vec{d}_{1}$ and the actual direction if we link directly $P_{e}$ and $P_{c}$. Figure 3 illustrates the $\Theta\left(P_{e}, P_{c}\right)$ term.

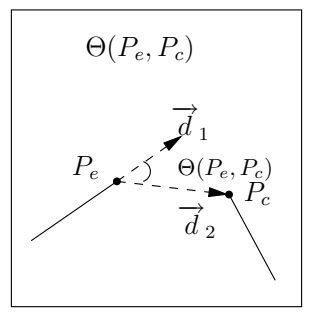

Fig. 3. $\Theta\left(P_{e}, P_{c}\right)$ term of Equation 2 
The Pseudocode 1.1 resumes the proposed algorithm.

Pseudocode 1. Proposed ridge linking algorithm.
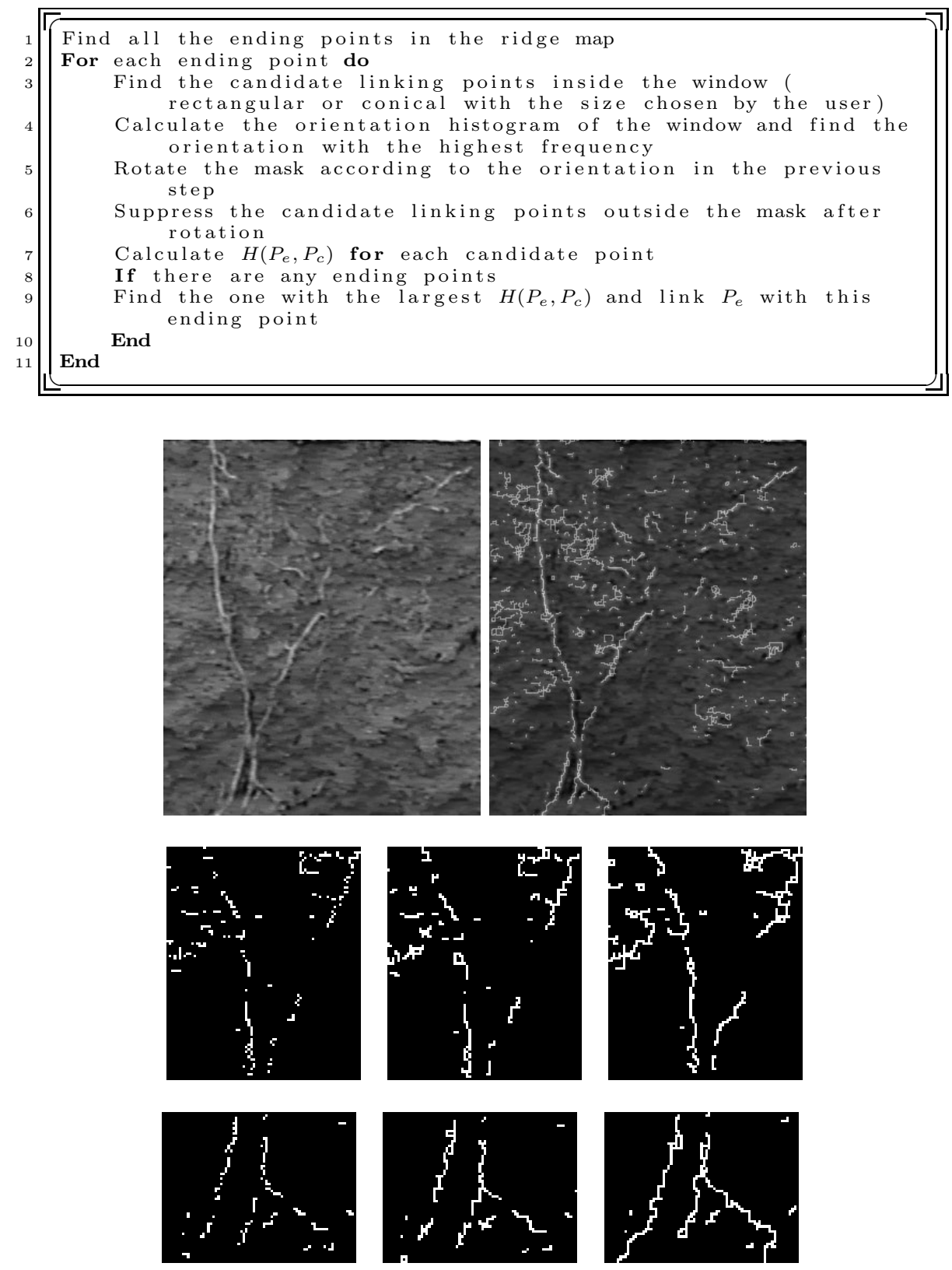

Fig. 4. From left to right and top to bottom: first row shows an original plant root image and the final linked ridge map superposed to it; second and third rows show, each one, a selected area from ridge map, linking result by [13] and linking result by our method. 


\section{$3 \quad$ Experiments}

In this section we show some experiments using the proposed algorithm in plant root images. The original edge linking algorithm proposed by [13] was also applied to these images. However, we use the ridges instead of the edges.

First of all, in order to show the improvement related to the rotation of the mask and the directional context of the image, we present, in Figures 4 and 5 , the application of our method on real images of plant roots. The first row shows the original image and the final linked ridge map superposed to it respectively. The second and third rows are organized, from left to right, as: selected area from the ridge map, linking result by [13 and linking result by our method. This kind of structure is well characterized by its directions and we can notice that our results are more connected, smoother and retain more the directional context of the structures in the image. Figure 6 presents one more example.

Besides, in order to confirm the improvements of the new algorithm, we applied it to 10 real plant root images, which are ground-truthed by an expert, and we calculated the True Positive Rate (TPR) and the False Positive Rate (FPR) in each case and for the original ridge map. The mean values are shown
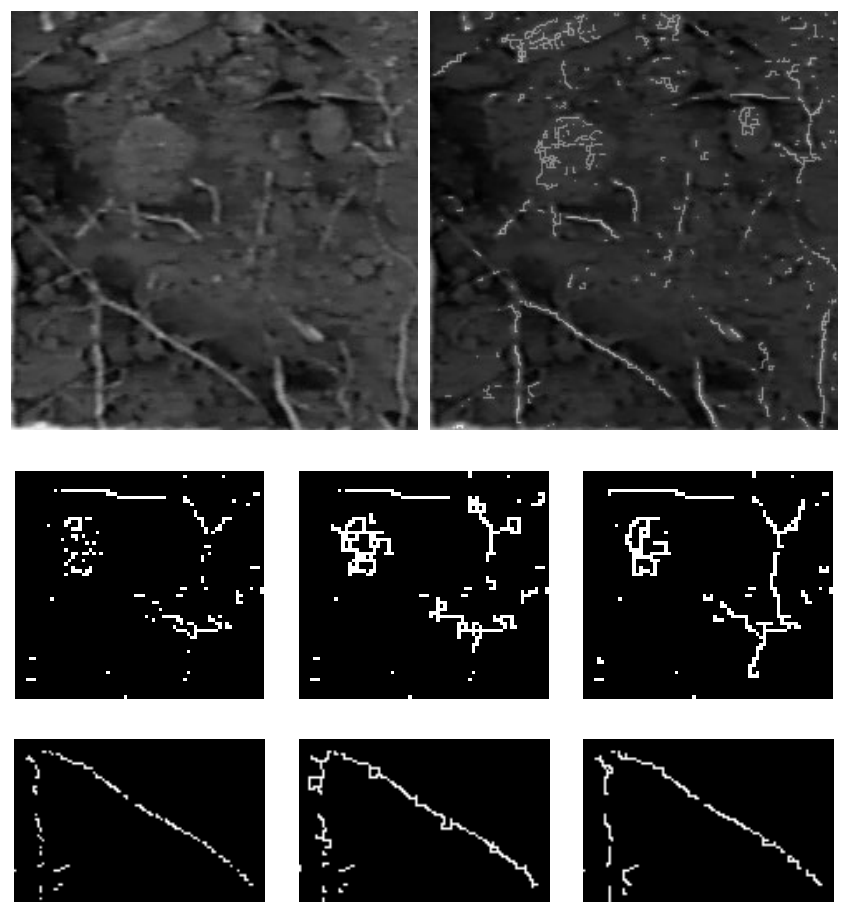

Fig. 5. From left to right and top to bottom: first row shows an original plant root image and the final linked ridge map superposed to it; second and third rows show, each one, a selected area from ridge map, linking result by [13] and linking result by our method 

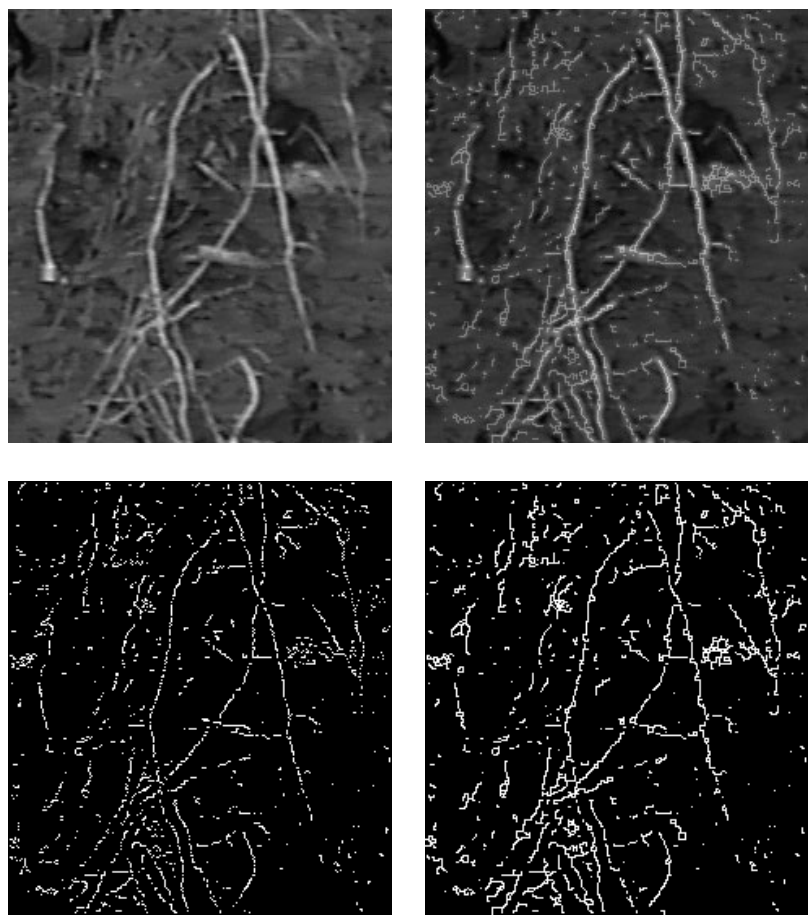

Fig. 6. From left to right and top to bottom: first row shows an original plant root image and the final linked ridge map superposed to it; second row show the ridge map and the linking result by the proposed method

Table 1. Mean values of TPR and FPR after the application of both algorithms to 10 real plant root images

\begin{tabular}{l|c|c|c}
\hline & \multicolumn{4}{|c}{$\begin{array}{c}\text { Measures } \\
\text { Classification rates }\end{array}$} & Ridge map & Original algorithm & Proposed algorithm \\
\hline \hline TPR & 0.67 & 0.71 & 0.77 \\
\hline FPR & 0.072 & 0.086 & 0.09 \\
\hline
\end{tabular}

in Table 1. One can see that our algorithm has a good improvement of TPR with an acceptable low increasing of the FPR. The average number of false positives is high because of the kind of image we are using. Some improvements must be done to the ridge detector to decrease the false alarms.

\section{Conclusions}

We presented a new ridge linking algorithm derived from methods of edge linking already existing in the literature. Our approach aims to make a more precise 
decision to connect ridge segments using an adaptive directional mask. Results showed that our algorithm leads to good connections which tends to maintain the good continuation of structures like plant roots. To the best of our knowledge, there is no linking method in the literature with this characteristic. Specific applications, mainly those that deal with structures characterized by its directions, can take advantages of our technique. This approach can help in the segmentation of thin and ramified structures. We also applied this technique in common images using the edges and we obtained good results.

\section{References}

1. Basak, J., Chanda, B., Dutta Majumder, D.: On edge and line linking with connectionist models. IEEE Transactions on Systems, Man and Cybernetics 24(3), 413-428 (1994)

2. Cook, G.W., Delp, E.J.: Multiresolution sequential edge linking. In: International Conference on Image Processing, vol. 1, p. 41 (1995)

3. Eichel, P.H., Delp, E.J.: Sequential edge detection in correlated random fields. In: Proceedings of the IEEE Computer Vision and Pattern Recognition Conference, pp. 15-21 (June 1985)

4. Eichel, P.H., Delp, E.J., Koral, K., Buda, A.J.: A method for a fully automatic definition of coronary arterial edges from cineangiograms. IEEE Transactions on Medical Imaging 7(4), 313-320 (1988)

5. Grigorescu, C., Petkov, N., Westenberg, M.A.: Contour and boundary detection improved by surround suppression of texture edges. Image Vision Comput. 22(8), 609-622 (2004)

6. Hajjar, A., Chen, T.: A vlsi architecture for real-time edge linking. IEEE Transactions on Pattern Analysis and Machine Intelligence 21(1), 89-94 (1999)

7. Li, J., Randall, J., Guan, L.: Perceptual image processing for digital edge linking. In: Canadian Conference on Electrical and Computer Engineering, IEEE CCECE 2003, vol. 2, pp. 1215-1218 (May 2003)

8. Miller, F.R., Maeda, J., Kubo, H.: Template based method of edge linking using a weighted decision. In: Proceedings of the IEEE/RSJ International Conference on Intelligent Robots and Systems, IROS 1993, vol. 3, pp. 1808-1815 (July 1993)

9. Sappa, A.D.: Unsupervised contour closure algorithm for range image edge-based segmentation. IEEE Transactions on Image Processing 15(2), 377-384 (2006)

10. Sappa, A.D., Vintimilla, B.X.: Cost-based closed-contour representations. Journal of Electronic Imaging 16(2), 023009/1-023009/9 (2007)

11. Shih, F.Y., Cheng, S.: Adaptive mathematical morphology for edge linking. Inf. Sci. Inf. Comput. Sci. 167(1-4), 9-21 (2004)

12. Stahl, J.S., Wang, S.: Edge grouping combining boundary and region information. IEEE Transactions on Image Processing 16(10), 2590-2606 (2007)

13. Wang, Z., Zhang, H.: Edge linking using geodesic distance and neighborhood information. In: IEEE/ASME International Conference on Advanced Intelligent Mechatronics, AIM 2008, pp. 151-155 (July 2008) 